\title{
Reducing Order and Inventory Variability under Stochastic Lead-time and Correlated Demand
}

\author{
(presented at the $6^{\text {th }}$ IESM Conference, October 2015, Seville, Spain) (c) $I^{4} e^{2} 2015$ \\ Xun Wang \\ Logistics System Dynamics Group, \\ Cardiff Business School \\ Colum Drive \\ Cardiff, UK \\ Email: wangx46@cardiff.ac.uk \\ Stephen M. Disney \\ Logistics System Dynamics Group, \\ Cardiff Business School \\ Colum Drive \\ Cardiff, UK \\ Email: disneysm@cardiff.ac.uk
}

\begin{abstract}
Logistic volatility is considered to be an important contributor to supply chain inefficiency. In this paper we investigate the amplification of order and inventory fluctuations in a state-space model with stochastic lead time, $\operatorname{ARMA}(p, q)$ demand and a proportional order-up-to policy. We derive the exact distribution functions for order and inventory. For i.i.d. Gaussian demand, we prove that the proportional outperforms the classical order-up-to policy in reducing inventory and order variances simultaneously. Numerical experiments are carried out to show the complex interaction between demand correlation and stochastic lead-time.
\end{abstract}

\section{INTRODUCTION}

We investigate the ability of the proportional order-up-to policy to reduce variance amplification under stochastic lead time. Variation in inventory systems is commonly generated by uncertainties in demand, supply, transportation, and manufacturing, and can be amplified by poorly designed inventory control mechanisms [1] [2]. Variance amplification in inventory systems, which can be understood as quadratic costs representing diseconomies of scale [3], pose a large threat to companies at the operational level. High order variance (a.k.a. the bullwhip effect) brings more uncertainty to the upstream supplier, and induces costs associated with production adjustment and capacity utilization. High inventory variance requires more safety stock to accommodate increased stock-out risk, reducing supply chain efficiency.

Uncertainty in logistics, i.e., stochastic shipping delays, is a major component of supply chain risks. In recent years, production and distribution systems have become increasingly global, exposing supply chains to more volatility than ever before. Global transportation modes, such as air, truck, rail and ocean freight, have long and variable lead times, due to external factors such as seasonality effects, security and customs delays and slow steaming. Uncertain lead-times sometimes trigger another effect called order crossover, when replenishments are received in a different sequence than they were ordered. Whilst these two concepts do not necessarily imply each other, a highly variable lead time often results in order crossover [4] [5].

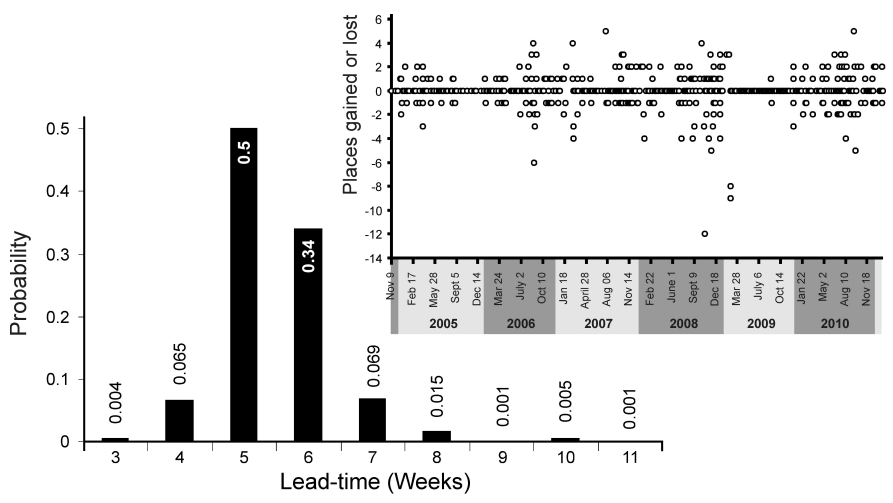

Fig. 1. Empirical evidence of global door-to-door shipping lead time from USA to China

Both stochastic lead time and order crossover are common in practice. Figure 1 contains data from a company who manufacturers products in the USA and ships them in China for assembly. The histogram shows statistics of the transportation time used for 827 containers by truck and ship between 2004 and 2011. We see the possible lead time ranges from 3 to 11 weeks. The mode of lead time is 5 weeks which has only a probability of 0.5 . In the upright sub-figure, the sequence of dispatches is compared to the sequence of the receipt. It shows the change of the position of an order in the dispatch and receiving sequences, from which we see a significant number of order crossovers.

Ref. [6] classified the research on stochastic lead times into three schools: The Hadley-Whitin School, which assumes that the probability of order crossover is so small that it can be totally ignored [7]. The Zipkin-Song School, which assumes that goods are transported sequentially so that order crossover cannot happen [4] [8]. And the Zalkind School, which takes order crossover into account and discovers that inventory cost as well as safety stock can be reduced by considering this effect [9] [10] [11]. Our model is of the Zalkind type since it allows order crossover. This kind of model is firstly introduced by [12] and later [13], in which 
they gave the correct expression for the distribution of the number of outstanding orders. Ref. [9] determined the optimal target inventory level to minimize total cost. Ref. [14] showed the importance of considering order crossover when setting safety stock. Ref. [10] pointed out that order crossover has a significant impact on inventory control and should not be ignored. The aims of these studies are either to derive (approximate) relevant distributions or to decide safety stock level parameters. Others have investigated the bullwhip effect with stochastic lead time, adopting the assumptions of i.i.d. demand and the order-up-to policy [15] [16].

The proportional order-up-to policy proved to be effective at smoothing the bullwhip effect, at a cost of amplified inventory variation [17] [18] [19] [20]. However, most studies on the bullwhip effect require at least a predictable, if not a constant, lead time; while existing research on stochastic lead time problems do not explicitly tackle the bullwhip problem. To fill these gaps, we answer two sequential problems: (i) How are orders and inventory distributed under stochastic lead-time and correlated demand? (ii) Can the proportional ordering policy help to reduce these variances?

Our research is descriptive rather than prescriptive in the sense that we seek to understand two known replenishment policiesthe order-up-to and proportional order-up-to policyrather than to seek optimal policies for a given objective function. That said, the order-up-to policy is known to be inventory cost optimal for constant lead-time or stochastic lead-time without crossover. We will show the proportional policy outperforms the order-up-to policy under a stochastic lead-time with order crossovers for quadratic objectives. Its optimality is beyond the scope of this paper. We note that the linear proportional policy: allows us to completely characterize the solution; is implementable in the real world [21]; and is easy to incorporate into enterprise resource planning systems.

The contribution of this research lies in the following aspects. Firstly, we derive the density functions of orders and inventory under the proportional order-up-to policy, stochastic lead time and general $\operatorname{ARMA}(p, q)$ demand using state space techniques. Secondly, we give a necessary condition for the optimality, based on which we find that the classical order-up-to policy is never optimal at minimizing inventory variance when order crossover is present. The most significant discovery is that the trade-off between order and inventory variability [17] may be broken by the proportional policy in an order crossover environment.

The paper is organized as follows. In Section 2 we introduce basic notation and models of the demand and ordering policies; Section 3 contains the main results, which include an exact approach to obtain the distribution of order and inventory, and conditions for the optimality of the order-up-to policy; in Section 4 we observe the impact of demand correlation, forecasting method and lead time uncertainty via numerical experiments. Concluding remarks are given in Section 5. Due to space limitation we omit the proofs of all the mathematical derivations, which will be available upon request.

\section{MOdELING THE DEMAND AND ORDERING POLICY}

We focus on a periodic review system where the system states are defined in the real field $\Re$. Lead time is a discrete i.i.d. process with an arbitrary distribution. Denote $\Sigma_{x y}(\tau)$ as the mutual correlation function between the random variables $x$ and $y$. Specifically, $\Sigma_{x x}(\tau)$ is the auto correlation function of $x$. When $\tau=0, \Sigma_{x x}(0)$ equals the variance of $x$. Sometimes we write this as $\Sigma_{x x}$ if no other confusion would occur. $E(x)$ or $\bar{d}$ is the expectation of $x$, and $x^{T}$ is the transpose of $x$. The notation of system variables is: $d$ for demand; $o$ for order; $i$ for inventory (on-hand); $w$ for work-in-process (WIP, inventoryon-order, total outstanding orders); ss for safety stock; $L$ is the lead-time; $L^{+}$and $L^{-}$are maximum and minimum leadtimes. $p_{l}$ represents the probability that the lead-time of an order is $l$, that is, an order made at time $t$ completes at time $t+l$. Other notation will be introduced when necessary.

\section{A. The demand process}

$\operatorname{An} \operatorname{ARMA}(p, q)$ demand can be formulated as a constant $\bar{d}$ plus a zero mean $\operatorname{ARMA}(p, q)$ process, $d_{t}=z_{t}+\bar{d}$, where

$$
z_{t}=\phi_{1} z_{t-1}+\cdots+\phi_{p} z_{t-p}-\theta_{1} \epsilon_{t-1}-\cdots-\theta_{q} \epsilon_{t-q}
$$

Here $\left\{\epsilon_{t}\right\}$ is a Gaussian white noise process. Let $m=$ $\max (p, q+1)$ and $\theta_{j}=0$ for $j=q+1, \ldots, m ; \phi_{j}=0$ for $j=p+1, \ldots, m$. Introduce another $m$-dimensional vector $y$ such that

$y_{j, t}=\phi_{j} z_{t-1}+\cdots+\phi_{m} z_{t+j-m-1}-\theta_{j} \epsilon_{t-1}-\cdots-\theta_{m} \epsilon_{t+j-m-1}$

$y_{j, t}$ is the $j$ th element of $y_{t}, j=1,2, \ldots, m$. With this definition we can transform the ARMA demand model into a canonical state space form

$$
y_{t}=A y_{t-1}+B \epsilon_{t}, \quad z_{t}=C y_{t}
$$

Here $A$ is an $m \times m$ left companion matrix with $A_{j 1}=\phi_{j}$ and $A_{j, j+1}=1 . B=\left(\begin{array}{lll}1-\theta_{1} & \cdots & -\theta_{m-1}\end{array}\right)^{T}$ and $C=$ $\left(\begin{array}{llll}1 & 0 & \cdots & 0\end{array}\right)$.

The auto correlation of $y$, the stochastic component of demand, satisfies

$$
\Sigma_{y y}=A \Sigma_{y y} A^{T}+B B^{T}
$$

The solution of (2) can be obtained by the Kronecker product:

$$
\operatorname{vec}\left(\Sigma_{y y}\right)=(I-A \otimes A)^{T} \operatorname{vec}\left(B B^{T}\right)
$$

in which $\otimes$ denotes the Kronecker (tensor) product of matrices and $\operatorname{vec}(\cdot)$ is the matrix column-wise stacking operation. $I$ is the identity matrix with appropriate dimension. Both the 
lead time distribution and demand correlation are assumed to be known in advance. In practice this can be realized by statistically analyzing historical data.

\section{B. The proportional order-up-to policy}

We study the proportional order-up-to policy, which can be formed by adding a proportional controller into the inventory position feedback loop of the order-up-to policy. This policy has been studied by [22] [23] [24] [17]. We start from the classic order-up-to policy under constant lead-time before introducing the proportional order-up-to policy under stochastic lead-time. We assume negative orders are allowed, which is known as the costless return assumption and is commonly seen in the literature [1].

The order-up-to policy can be written as

$$
o_{t}=S_{t}-I P_{t}
$$

where $S_{t}$ is the time-varying order-up-to level equal to the sum of the lead-time demand forecast and safety stock, $S_{t}=$ $\hat{D}_{t}+s s . s s$ is a constant related to the variance of the forecast error over the lead time and review period and the desired inventory service level. Under constant lead-time $L, \hat{D}_{t}=$ $\sum_{k=1}^{L} \hat{d}_{t, k}$, where $\hat{d}_{t, k}$ is the $k$-period ahead demand forecast made at time $t$. The order-up-to policy can be rewritten as

$$
o_{t}=\hat{d}_{t, L}+\left(s s+\hat{w}_{t}-i_{t}-w_{t}\right)
$$

where $\hat{w}_{t}=\sum_{k=1}^{L-1} \hat{d}_{t, k}$ and $w_{t}=\sum_{k=1}^{L-1} \hat{d}_{o_{t-k}}$. Since $E(i)=s s$ and $E(w)=E(\hat{w})=(L-1) \bar{d}$, ss and $\hat{w}_{t}$ serve as the target inventory level and the (time-varying) target WIP level respectively.

The proportional order-up-to policy is formed by adding a proportional controller $1-\lambda$ to the second term (feedback term), $\lambda \in(-1,1)$ :

$$
o_{t}=\hat{d}_{t, L}+(1-\lambda)\left(s s+\hat{w}_{t}-i_{t}-w_{t}\right)
$$

In other words, the order quantity equals the $L$-step-ahead demand forecast plus a fraction of the discrepancy between target and actual inventory positions. Since the lead-time is a discrete i.i.d. random variable with known distribution, the demand forecast and target WIP level are calculated as averages over all possible lead-time values:

$$
\hat{d}_{t, L}=\sum_{k=L^{-}}^{L^{+}} p_{k} \hat{d}_{t, k}, \hat{w}_{t}=\sum_{l=L^{-}}^{L^{+}} p_{l} \sum_{k=1}^{l-1} \hat{d}_{t, k}
$$

Rearranging (4) yields

$$
o_{t}=f_{t}+(1-\lambda)\left(s s-i_{t}-w_{t}\right)
$$

where $f_{t}$ is the forecast term

$$
f_{t}=\sum_{k=L^{-}}^{L^{+}} p_{k} \hat{d}_{t, k}+(1-\lambda) \sum_{l=L^{-}}^{L^{+}} p_{l} \sum_{k=1}^{l-1} \hat{d}_{t, k}
$$

\section{The MMSE forecasting policy}

We assume the firm adopts a minimum mean squared error (MMSE) forecast which minimizes the squared forecast error for a given ARMA process, as its name suggests. The MMSE $k$-step ahead forecast for $y$ can be calculated in state space form as

$$
\hat{y}_{t, k}=A^{k} y_{t}
$$

where $A^{k}$ is the matrix $A$ raised to its $k$ th power. The $k$ step ahead forecast of $z$ and $d$ are then simply $\hat{d}_{t, k}=\hat{z}_{t, k}$ and $\hat{z}_{t, k}=C \hat{y}_{t, k}$. From (8) we see that the MMSE forecast is linear, which enables us to rewrite $f_{t}$ as a linear function of $y_{t}$, i.e., $f_{t}=y_{t}+(\bar{L}-\lambda \bar{L}-\lambda) \bar{d}$. The second term is the expectation of (7) as $E(y)=0 . F$ is the forecasting vector which equals

$$
F=\sum_{k=L^{-}}^{L^{+}} p_{k} C A^{k}+(1-\lambda) \sum_{l=L^{-}}^{L^{+}} p_{l} \sum_{k=1}^{l-1} C A^{k}
$$

The variance of forecast takes the quadratic form:

$$
\Sigma_{f f}=F \Sigma_{y y} F^{T}
$$

\section{The balance equations}

The balance equations for inventory and WIP are

$$
i_{t}=i_{t-1}+o_{t-l}-d_{t}, w_{t}=w_{t-1}+o_{t-1}-o_{t-l}
$$

We also have

$$
E(o)=\bar{d}, E(I P)=s s+(\bar{L}-1) \bar{d}
$$

REMARK 1. The Principle of Separation [25] asserts that the optimal stochastic control problem can be separated to an optimal forecasting problem and a deterministic optimal control problem based on the forecast. This assists in understanding the structure of (6).

REMARK 2. The proportional order-up-to policy given by (6) and (7) together generalizes the classical order-up-to policy by adding a proportional feedback controller $1-\lambda$ and taking into account the stochastic lead time. When $\lambda=0$, the proportional order-up-to policy degenerates into the classical order-up-to policy. However for the purpose of clarification we unanimously refer to the proportional order-up-to policy as settings where $\lambda \neq 0$ in the remaining text.

\section{DISTRIBUTIONS OF ORDERS AND INVENTORY}

\section{A. Revisiting the constant lead-time case}

For illustration purposes, we will first introduce some wellestablished results showing the trade-off between order and inventory variance under constant lead-time. The demand is assumed to be i.i.d. but the trade-off also exists under correlated demand.

Lemma 3.1: 
(Trade-off between order and inventory variance)

Assuming i.i.d. demand, constant lead-time $L$ and proportional order-up-to policy 6 , the order and inventory variances are

$$
\begin{gathered}
\Sigma_{o o}=\frac{1-\lambda}{1+\lambda} \Sigma_{d d} \\
\Sigma_{i i}=\left(\frac{\lambda^{2}}{1-\lambda^{2}}+L\right) \Sigma_{d d}
\end{gathered}
$$

The following observations can be made from Lemma 3.1. (i) The optimal $\lambda$ for minimizing $\Sigma_{o o}$ and $\Sigma_{i i}$ are 1 and 0 respectively. In other words, the order-up-to policy minimizes $\Sigma_{i i}$; proportional order-up-to policy is able to reduce $\Sigma_{o o}$ at a cost of increased $\Sigma_{i i}$. (ii) $\Sigma_{o o}$ is independent of lead-time while $\Sigma_{i i}$ is not. (iii) Both $\Sigma_{o o}$ and $\Sigma_{i i}$ are independent with average demand.

\section{B. Covariance functions of the orders}

The following proposition gives the auto- and mutualcovariance function between $y$ and $o$ which is required to calculate the distribution of inventory.

Proposition 3.2:

(Covariance of orders)

For demand process given by (1), the ordering policy by (6) and the inventory evolution by (9), we have:

Covariance between the demand and orders:

$$
\begin{array}{r}
\Sigma_{y o}=(I-\lambda A)^{-1}(I-A) \Sigma_{y y} F^{T}+ \\
(1-\lambda)(I-\lambda A)^{-1} \Sigma_{y y} C^{T}
\end{array}
$$

Autocorrelation function between demand and orders:

$$
\Sigma_{y o}(\tau)=A^{\tau} \Sigma_{y o}(0)
$$

Variance of the orders:

$$
\begin{aligned}
\Sigma_{o o}= & \frac{2}{1+\lambda} F(I-\lambda A)^{-1}(I-A) \Sigma_{y y} F^{T}+ \\
& \frac{2 \lambda}{1+\lambda} F(A-I)(I-\lambda A)^{-1} \Sigma_{y y} C^{T}+ \\
& \frac{2}{1+\lambda} C(I-\lambda A)^{-1}(I-A) \Sigma_{y y} F^{T}+ \\
& \frac{1-\lambda}{1+\lambda} C(I+\lambda A)(I-\lambda A)^{-1} \Sigma_{y y} C^{T}
\end{aligned}
$$

Autocorrelation function of orders:

$\Sigma_{o o}(\tau)=[F A+(1-\lambda) C A-F] \Sigma_{y o}(\tau-1)+\lambda \Sigma_{o o}(\tau-1)$

When demand is normally distributed, the orders are also normally distributed with the density function $\psi_{o}$ :

$$
\psi_{o}=\varphi\left(\bar{d}, \Sigma_{o o}\right)
$$

$\varphi\left(\mu, \sigma^{2}\right)$ is the probability density function (PDF) of a normal distribution with mean $\mu$ and variance $\sigma^{2}$. The average of ordering quantity is not affected by the stochastic leadtime. However the stochastic lead-time affects the variance of orders through the forecasting vector $F .3 .2$ not only gives the variance of orders, but also the auto/mutual covariance functions between $y$ and $o$ which is essential in calculating the inventory variance. In the rest of the paper the terms and order variance and bullwhip will be used interchangeably.

\section{Distribution of inventory under stochastic lead-time}

To calculate the inventory distribution we must first explain how it is affected by the stochastic lead time in terms of the outstanding order status. Under a fixed lead time scenario, whether $o_{t-\tau}$ is outstanding at time $t$ is deterministic. However, if lead time is stochastic then this status becomes probabilistic. Specifically the outstanding status of $o_{t-1}, o_{t-2}, \ldots, o_{t-L^{+}+1}$ determines the distribution of $w_{t}$ and $i_{t}$. It is convenient to introduce a binary row vector $\xi$ with $L^{+}-1$ elements to express whether orders $o_{t-1}, o_{t-2}, \ldots, o_{t-L^{+}+1}$ are outstanding or fulfilled time $t$. The $k$ th element of $\xi, \xi(k)=1$ means the order placed at time $t-k$ is outstanding at $t$ and $\xi(k)=0$ means the order placed at $t-k$ is completed at $t . k=1,2, \ldots, L^{+}-1$. We name $\xi$ the vector of outstanding status or completion status. It is worth noting that for any $t$, orders made before (and including) $t-L^{+}$are fulfilled, i.e., $\xi(k)=0$ for $k>t-L^{+}$; orders made after (and excluding) $t-L^{-}$are outstanding, i.e., $\xi(k)=1$ for $k>t-L^{-}$. So $L^{+}-1$ dimensions suffice to describe the current outstanding order status, in which $L^{+}-L^{-}$elements are stochastic and $L^{-}-1$ elements are deterministic and equal to one. Since each element in $\xi$ is Boolean, it has $2^{L^{+}-L^{-}}$possible realizations, which we denote as $\xi_{j}$. Here the subscript $j$ indexes all possible realizations of vector $\xi$, which should not be confused with $\xi(k)$, the $k$ th element of $\xi$.

The basic idea here is that in the long term, the WIP (and inventory) process can be seen as a composition of $2^{L^{+}-L^{-}}$ sub-processes, which are conditional on the outstanding status. In every period of each one of the sub-processes, the outstanding status of orders is identical and can be represented by $\xi$. In other words, all the elements in each sub-process are stationary and normally distributed. After identifying the characteristics of the sub-processes which are mutually independent (because lead-time is independently distributed), we can derive the distribution function of the full process. For each sub-process, the variance of WIP, inventory and the covariance between order and WIP are dependent on $\xi$ and will be denoted as $\Sigma_{w w}(\tau ; \xi), \Sigma_{i i}(\tau ; \xi)$ and $\Sigma_{o w}(\tau ; \xi)$ from now on.

EXAMPLE. Consider the lead time distribution: $p_{k}=1 / 3, k=1,2,3$. The second line in Table $\mathrm{I}$ is a realization of the stochastic lead time for each order over 10 periods. As $L^{+}=3$ we start investigation from period 3. Since the lead time for $o_{1}$ and $o_{2}$ are both 3, at period 3 they are both outstanding. At period 4, $o_{1}$ has arrived and so does $o_{3}$, which has a lead time of 1 . The only 
TABLE I

EXAMPLE OF STOCHASTIC LEAD TIME AND OUTSTANDING ORDERS

\begin{tabular}{|c|c|c|c|c|c|c|c|c|c|c|}
\hline Period & 1 & 2 & 3 & 4 & 5 & 6 & 7 & 8 & 9 & 10 \\
\hline Order lead-time & 3 & 3 & 1 & 3 & 2 & 1 & 1 & 3 & 1 & 2 \\
\hline Outstanding orders & - & - & $\begin{array}{c}o_{t-2} \\
o_{t-1}\end{array}$ & $o_{t-2}$ & $o_{t-1}$ & $\begin{array}{c}o_{t-2} \\
o_{t-1}\end{array}$ & None & None & $o_{t-1}$ & $o_{t-2}$ \\
\hline Outstanding status & - & - & $(1,1)$ & $(1,0)$ & $(0,1)$ & $(1,1)$ & $(0,0)$ & $(0,0)$ & $(0,1)$ & $(1,0)$ \\
\hline
\end{tabular}

outstanding order would be $\mathrm{O}_{2}$. At period $5, \mathrm{O}_{2}$ has arrived and $o_{4}$ is outstanding. This constitutes the third line of Table I.

The WIP with the same outstanding orders are identically distributed, e.g., periods 3 and 6 , periods 4 and 10, period 5 and 9 , periods 7 and 8 . Thus there are several sub-processes ( 4 in this example as $2^{3-1}=4$ ) in the WIP process. Each of the sub-processes is characterized by an outstanding status vector $((1,1),(1,0),(0,1)$ and $(0,0))$, shown in the last line of Table I. Since the distribution of inventory is the convolution of $f_{t}, o_{t}$ and $w_{t}$, and both $f_{t}, o_{t}$ are independent of the lead time realization, the inventory process can also likewise be decomposed into several sub-processes.

We use $p(\xi)$ to denote the probability of $\xi$, which is also the proportion of the sub-process characterized by $\xi$ in the entire process. Ref. [9] offered a simple approach to calculate $p(\xi)$ :

$$
p(\xi)=\prod_{k=1}^{L^{+}-1}\left\{[1-\xi(k)] \Psi_{L}(k)+\xi(k) \bar{\Psi}_{L}(k)\right\}
$$

$\Psi_{L}(\cdot)$ is the cumulative distribution function (CDF) and $\bar{\Psi}_{L}(\cdot)=1-\Psi_{L}(\cdot)$ is the complementary CDF of the lead time.

Work-in-process at time $t$ equals to the sum of all outstanding orders at time $t$, i.e.,

$$
w_{t}(\xi)=\sum_{k=1}^{L^{+}-1} \xi(k) o_{t-k}
$$

The above equation shows that WIP is a random sum of random variables under ARMA demand and the proportional order-up-to policy, except that these random variables (the orders) are inter-correlated. Thus, for expectations of WIP and inventory sub-processes with respect to order completion status and safety stock, we have

$$
\begin{aligned}
E(w ; \xi) & =\sum_{k=1}^{L^{+}-1} \xi(k) \bar{d}=\xi \mathbf{1} \bar{d} \\
E(i ; \xi, s s) & =s s+(\bar{L}-\xi \mathbf{1}-1) \bar{d}
\end{aligned}
$$

$\mathbf{1}$ is an unit column vector. $\xi \mathbf{1}$ is the number of outstanding orders under $\xi$. Note for different $\xi$, the number of outstanding orders can be the same. We show from the above analysis that the WIP and inventory distributions are summations of appropriately weighted normal distributions:

$$
\begin{aligned}
E(w ; \xi) & =\sum_{k=1}^{L^{+}-1} \xi(k) \bar{d}=\xi \mathbf{1} \bar{d} \\
E(i ; \xi, s s) & =s s+(\bar{L}-\xi \mathbf{1}-1) \bar{d}
\end{aligned}
$$

We are now ready to calculate the inventory variance.

Proposition 3.3:

(Variance of inventory)

The variance of inventory is given by

$$
\begin{aligned}
\Sigma_{i i} & =\frac{\Sigma_{f f}+\Sigma_{o o}-2 F \Sigma_{y o}}{(1-\lambda)^{2}} \\
& +\sum_{k=1}^{L^{+}-1} \beta_{k}\left[\Sigma_{o o}+2 \frac{\Sigma_{o o}(k)-\Sigma_{y o}(k)}{1-\lambda}\right] \\
& +\sum_{k=1}^{L^{+}-2} \gamma_{k} \Sigma_{o o}(k)+\bar{d}^{2} \Sigma_{N N}
\end{aligned}
$$

where

$$
\begin{gathered}
\beta_{k}=\bar{\Psi}_{L}(k) \\
\gamma_{k}=2 \sum_{j=1}^{L^{+}-k-1} \bar{\Psi}_{L}(j) \bar{\Psi}_{L}(j+k) \\
\Sigma_{N N}=\sum_{j>k} p\left(\xi_{j}\right) p\left(\xi_{k}\right)\left(\xi_{j} \mathbf{1}-\xi_{k} \mathbf{1}\right)^{2}
\end{gathered}
$$

The first term of (16) equals the inventory variance when $L=1$. The last term equals the inventory variance when the demand is constant, i.e., $d \equiv \bar{d}$. It can be seen that larger mean demands increase the inventory variance under stochastic lead times. This is contrary to the deterministic lead time case that the demand level does not affect the inventory variance. Moreover, since the inventory distribution $\psi_{i}$ is a summation of several normal distributions, it does not have to be, and almost never is, normally distributed. It is a multi-modal distribution as long as the lead time is random and $\bar{d}$ is non-zero.

The above approach to calculate inventory variance needs the lead time to be bounded, which is practically plausible. If the lead time follows an unbounded theoretical distribution, 
e.g., Poisson or compound Poisson, it could pose extra difficulties in computation. As pointed out by [10] this would require calculating an infinite sum of random variables. However, for any desired accuracy, our approach can always be extended to generate a satisfactory approximation.

\section{Optimization of the feedback controller}

In this section we give a necessary condition for the orderup-to policy $(\lambda=0)$ to be optimal at reducing $\Sigma_{i i}$. The condition is based on the optimality condition of the first derivative, . For ARMA demand and stochastic lead time, we have the following proposition:

\section{Proposition 3.4:}

(Order-up-to sub-optimality) Under i.i.d. demand, MMSE forecast and order crossover, the order-up-to policy is not optimal in reducing $\Sigma_{i i}$.

Proposition 3.4 contradicts Lemma 3.1 when order crossover is present. In all the inventory sub-processes characterized by the vector $\xi$, those which represent order crossover (when there is at least a 1 on the left side of a 0 ) all can be minimized by a $\lambda>0$. Those without crossover are minimized by $\lambda=0$. Therefore the full inventory process, which is constituted by all the sub-processes, is minimized by a $\lambda>0$.

\section{NUMERICAL EXPERIMENTS}

In this section we conduct numerical experiments to demonstrate our analytical results above, and to show the mixed impact of stochastic lead-time, order crossover and demand correlation. In the experiments the expected demand is 5 units per period. In Table II and Table III we compare inventory variance and bullwhip under the order-up-to policy and optimized proportional order-up-to policy. Demand follows an i.i.d. process in Table II, and an $\mathrm{AR}(2)$ process with $\phi_{1}=0.6$ and $\phi_{1}=-0.9$ in Table III. We consider 10 different lead-time distributions (see Figure 2). There are both noncrossover (i, ii) and crossover (iii-x) scenarios. In the crossover scenarios we further investigate several distributions where lead-time variability gradually increases. We start with low variability distributions (iii, vii), to higher variability (iv, viii), uniform (v, ix) and then two-point distributions (vi, x). Once we have obtained the expressions for inventory variance, the optimal feedback parameter $1-\lambda^{*}$ can be solved numerically. We observe the values of optimal controllers in minimizing inventory variance. Then we compare the inventory variance and the bullwhip, in order-up-to and optimized proportional order-up-to scenarios.

First of all, we can observe that the stochastic lead-time affects the optimal controller selection only when there is order crossover. When crossover is not present (cases i and ii), the optimal controller to minimize inventory variance is unity and the proportional order-up-to policy does not reduce either the inventory variance or the bullwhip effect. However, for the order crossover scenarios (iii-x), an optimized proportional order-up-to policy is able to mitigate the bullwhip effect significantly while mildly reducing the inventory variance. This implies that the trade-off between the bullwhip effect

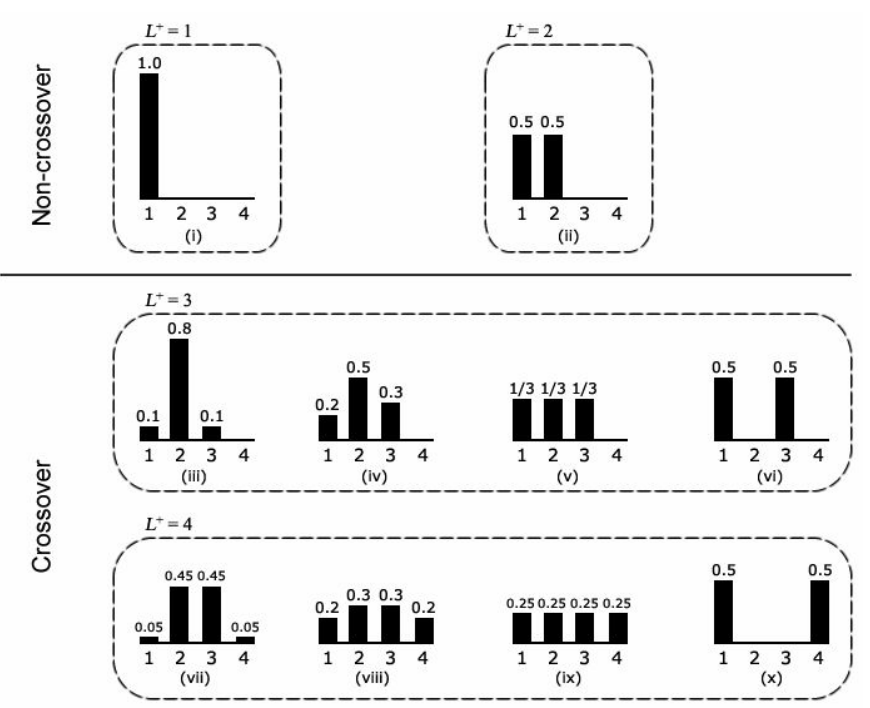

Fig. 2. Lead-time distribution scenarios in the numerical experiment

and inventory variability can be broken by the proportional policy in order crossover scenarios. The reduction in bullwhip is more considerable when lead-time volatility is high, e.g., when it follows a two-point distribution (cases vi and $\mathrm{x}$ ). When the lead-time is less variable, the optimal controller gets closer to unity, and the benefit of proportional policy becomes smaller.

For illustration purposes, we have plotted the inventory variance and bullwhip w.r.t. $\lambda$ for case vi (see Figure 3, where the scales are adjusted) under i.i.d. demand. The existence of minimal $\Sigma_{i i}$ and the monotonicity of $\Sigma_{o o}$ can be clearly seen. The inventory variance reaches minimum while $\lambda=0.13$ whereas the bullwhip decreases with $\lambda$. So setting $\lambda$ to 0.13 reduces both inventory variance and bullwhip (compared to $\lambda=0$ ). As $\lambda$ increases, the bullwhip will continue to decrease; however this comes with increased inventory variance (e.g. when $\lambda>0.26$ ).

The benefit of proportional policy on order and inventory variance is obviously asymmetric. This can be explained as follows. The inventory distribution is multimodal with modes separated by $\bar{d}$. While $1-\lambda^{*}$ reduces the variance of each mode, it has no effect on the mode separation. Therefore when we determine the variance of the whole inventory distribution, it is dominated by $\bar{d}$. The order distribution is unaffected by $\bar{d}$ and is a unimodal normal distribution; because of this the influence of $1-\lambda^{*}$ on order variance is more substantial.

When the demand follows an autoregressive process, similar observations can be made (Table III), the most obvious difference being that the demand auto-correlation has a distortion effect on the relationship between lead-time volatility and the benefit of the proportional policy. Sometimes 
TABLE II

OPTIMAL FEEDBACK CONTROLLER AND SYSTEM VARIABILITY WITH DIFFERENT LEAD TIME DISTRIBUTIONS AND I.I.D. DEMAND

\begin{tabular}{|c|c|c|c|c|c|c|c|c|c|}
\hline \multicolumn{3}{|c|}{ Lead-time distribution } & \multicolumn{2}{|c|}{ OUT $(1-\lambda=1)$} & \multicolumn{3}{|c|}{ POUT } & \multirow{2}{*}{$\begin{array}{l}\% \Sigma_{i i} \\
\text { Reduction }\end{array}$} & \multirow{2}{*}{$\begin{array}{l}\% \Sigma_{o o} \\
\text { Reduction }\end{array}$} \\
\hline No. & & Description & $\Sigma_{i i}$ & $\Sigma_{o o}$ & $1-\lambda^{*}$ & $\Sigma_{i i}$ & $\Sigma_{o o}$ & & \\
\hline i & $L^{-}=L^{+}=1$ & $p_{1}=1$ & 1 & 1 & 1 & 1 & 1 & 0.00 & 0.00 \\
\hline ii & $L^{-}=1, L^{+}=2$ & $p_{1}=0.5, p_{2}=0.5$ & 7.75 & 1 & 1 & 7.75 & 1 & 0.00 & 0.00 \\
\hline iii & \multirow{4}{*}{$L^{-}=1, L^{+}=3$} & $p_{1}=0.1, p_{2}=0.8, p_{3}=0.1$ & 6.50 & 1 & 0.99 & 6.50 & 0.98 & 0.00 & 2.00 \\
\hline iv & & $p_{1}=0.2, p_{2}=0.5, p_{3}=0.3$ & 11.35 & 1 & 0.95 & 11.35 & 0.91 & 0.00 & 9.00 \\
\hline $\mathrm{v}$ & & $p_{l}=1 / 3, l=1,2,3$ & 13.11 & 1 & 0.92 & 13.10 & 0.85 & 0.08 & 15.00 \\
\hline vi & & $p_{1}=0.5, p_{3}=0.5$ & 14.50 & 1 & 0.87 & 14.47 & 0.76 & 0.21 & 24.00 \\
\hline vii & \multirow{4}{*}{$L^{-}=1, L^{+}=4$} & $\begin{array}{l}p_{1}=0.05, p_{2}=0.45 \\
p_{3}=0.45, p_{4}=0.05\end{array}$ & 11.12 & 1 & 0.96 & 11.12 & 0.92 & 0.00 & 8.00 \\
\hline viii & & $\begin{array}{l}p_{1}=0.2, p_{2}=0.3 \\
p_{3}=0.3, p_{4}=0.2\end{array}$ & 16.75 & 1 & 0.88 & 16.73 & 0.78 & 0.12 & 22.00 \\
\hline ix & & $p_{l}=1 / 4, l=1,2,3,4$ & 18.13 & 1 & 0.86 & 18.09 & 0.75 & 0.22 & 25.00 \\
\hline $\mathrm{x}$ & & $p_{1}=0.5, p_{4}=0.5$ & 21.25 & 1 & 0.79 & 21.14 & 0.65 & 0.52 & 35.00 \\
\hline
\end{tabular}

TABLE III

OPTIMAL FEEDBACK CONTROLLER AND SYSTEM VARIABILITY WITH DIFFERENT LEAD TIME DISTRIBUTIONS AND AR(2) DEMAND

\begin{tabular}{|c|c|c|c|c|c|c|c|c|c|}
\hline \multicolumn{3}{|c|}{ Lead-time distribution } & \multicolumn{2}{|c|}{ OUT $(1-\lambda=1)$} & \multicolumn{3}{|c|}{ POUT } & \multirow{2}{*}{$\begin{array}{l}\% \Sigma_{i i} \\
\text { Reduction }\end{array}$} & \multirow{2}{*}{$\begin{array}{l}\% \Sigma_{o o} \\
\text { Reduction }\end{array}$} \\
\hline No. & & Description & $\Sigma_{i i}$ & $\Sigma_{o o}$ & $1-\lambda^{*}$ & $\Sigma_{i i}$ & $\Sigma_{o o}$ & & \\
\hline $\mathrm{i}$ & $L^{-}=L^{+}=1$ & $p_{1}=1$ & 1 & 7.05 & 1 & 1 & 7.05 & 0.00 & 0.00 \\
\hline ii & $L^{-}=1, L^{+}=2$ & $p_{1}=0.5, p_{2}=0.5$ & 9.65 & 7.42 & 1 & 9.65 & 7.42 & 0.00 & 0.00 \\
\hline iii & \multirow{4}{*}{$L^{-}=1, L^{+}=3$} & $p_{1}=0.1, p_{2}=0.8, p_{3}=0.1$ & 8.73 & 4.19 & 0.99 & 8.73 & 4.13 & 0.00 & 1.43 \\
\hline iv & & $p_{1}=0.2, p_{2}=0.5, p_{3}=0.3$ & 14.43 & 2.64 & 0.94 & 14.42 & 2.43 & 0.07 & 7.95 \\
\hline $\mathrm{v}$ & & $p_{l}=1 / 3, l=1,2,3$ & 16.50 & 2.16 & 0.91 & 16.48 & 1.87 & 0.12 & 13.43 \\
\hline vi & & $p_{1}=0.5, p_{3}=0.5$ & 18.37 & 1.24 & 0.85 & 18.32 & 0.92 & 0.27 & 25.81 \\
\hline vii & \multirow{4}{*}{$L^{-}=1, L^{+}=4$} & $\begin{array}{l}p_{1}=0.05, p_{2}=0.45 \\
p_{3}=0.45, p_{4}=0.05\end{array}$ & 14.15 & 2.26 & 0.95 & 14.15 & 2.15 & 0.00 & 4.87 \\
\hline viii & & $\begin{array}{l}p_{1}=0.2, p_{2}=0.3 \\
p_{3}=0.3, p_{4}=0.2\end{array}$ & 20.51 & 1.05 & 0.86 & 20.48 & 0.83 & 0.15 & 20.95 \\
\hline ix & & $p_{l}=1 / 4, l=1,2,3,4$ & 21.98 & 0.83 & 0.85 & 21.94 & 0.60 & 0.18 & 27.71 \\
\hline $\mathrm{x}$ & & $p_{1}=0.5, p_{4}=0.5$ & 24.45 & 1.13 & 0.79 & 24.42 & 0.94 & 0.12 & 16.81 \\
\hline
\end{tabular}

(e.g. in cases vii and viii) the proportional policy is more effective of reducing bullwhip and inventory under a less volatile lead-time distribution. Another effect of demand correlation is that the lead-time distribution now affects the bullwhip effect via the need for forecasting lead-time demand. As a consequence, it is possible that high lead-time variability can actually reduce bullwhip.

To further investigate the mixed effect of correlated demand and stochastic lead-time, we have calculated the inventory variance and bullwhip when demand is a first order autoregressive process AR(1) (See Figure 4). The correlation is either positive $(\phi=0.6)$ or negative $(\phi=-0.9)$. The lead-time follows a two-point or uniform lead-time distribution where $L^{-}$is set to be 1 and $L^{+}$varies from 2 to 10. Sub-figures (a) and (c) show the performance of the order-up-to policy when $=0$, and (b) and (d) show the optimized proportional order-up-to policy when $\lambda=\lambda^{*}$. From (a) and (b), we see that the inventory variance is strongly influenced by the lead-time variance (which is dominated by $L^{+}$) for both the two-point and the uniform lead-time distributions. Positive demand correlation leads to 


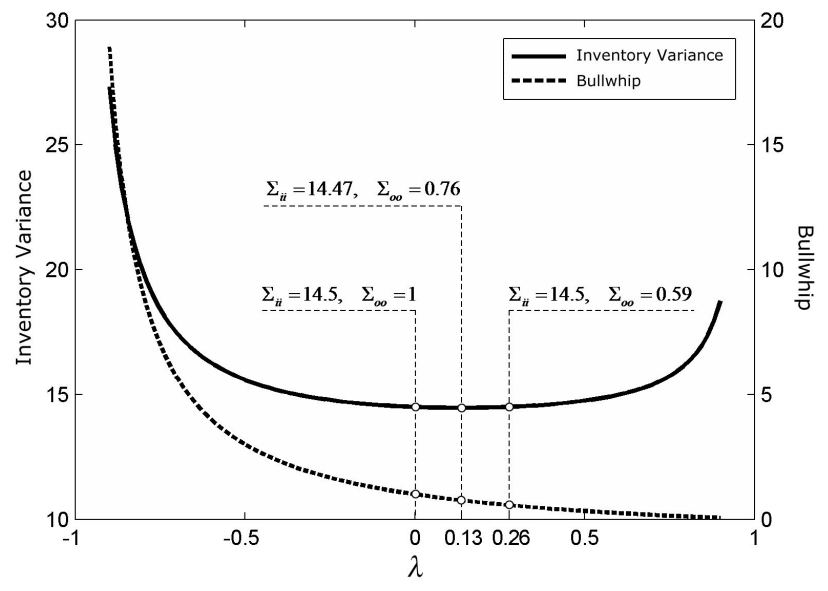

Fig. 3. Inventory variance and bullwhip in Case vi
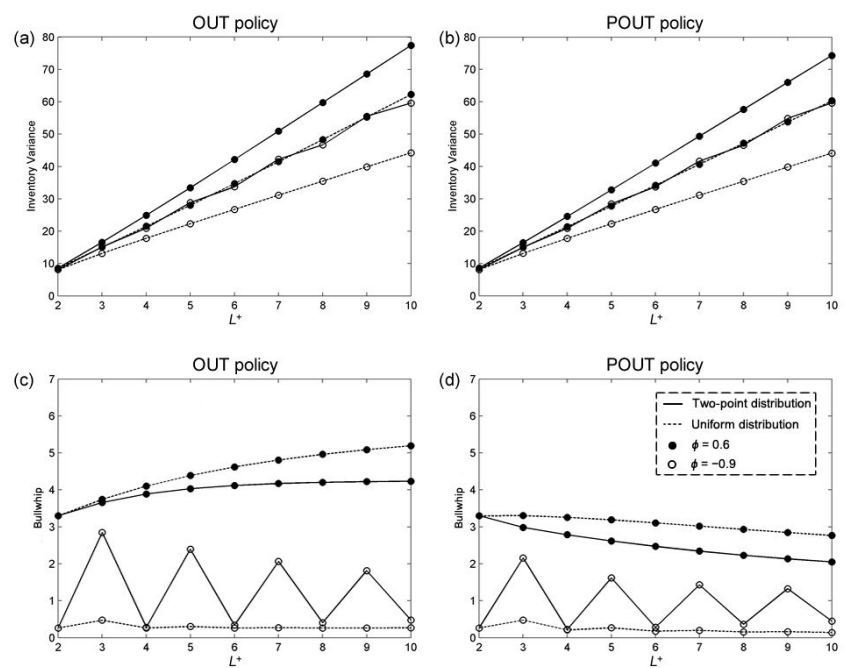

Fig. 4. Inventory and order variances when demand is an $\mathrm{AR}(1)$ process

higher inventory variance, and negative correlation creates an odd-even effect between lead-time and inventory variance (which is somewhat masked by the trend).

(c) and (d) show the corresponding bullwhip values under the order-up-to and proportional order-up-to policies, using the same $\lambda$ values in (a) and (b). The impact of the stochastic lead-time on order variance is not as decisive as on inventory variance, due to the fact that the lead-time distribution only affects the forecast of lead-time demand in the order calculation (6). Comparing the two-point and uniform lead-time distributions when demand is positively correlated, we see that low lead-time variance actually causes high order variance. Additionally, positive correlation still increases the order variance, and the fluctuation induced by negative correlation is more obvious. Most importantly, notice again that a large reduction of order variance can be achieved by optimizing the inventory variance, and that such reduction increases with the lead-time variability and the correlation coefficient.

Figure 5 illustrates how $\lambda^{*}$ changes with $L^{+}$when the leadtime follows a uniform distribution under different demand correlation. Again we see that the order-up-to policy is optimal when $L^{+}=1$ and $L^{+}=2$. When $L^{+}>2$, generally $\lambda^{*}$ increases with lead-time variability, which is especially true when demand is i.i.d. or positively correlated. This explains why the reduction of bullwhip is more significant for high lead-time variability and positive demand correlation. Under negatively correlated demand, the optimal $\lambda$ shows a periodic oscillating pattern along with an increasing trend. Under the more complicated $\operatorname{AR}(2)$ demand, the oscillation is less pronounced and has a low frequency but still can be seen, whereas the increasing trend persists. Moreover we can notice that under some correlated demand, (for example when $\phi=0.9$ and $L^{+}=3$ ). When $\lambda^{*}<0$ the tradeoff between bullwhip and inventory variance is restored, i.e., reducing inventory variance will increase bullwhip. However extensive calculations suggest that this effect rarely happens.

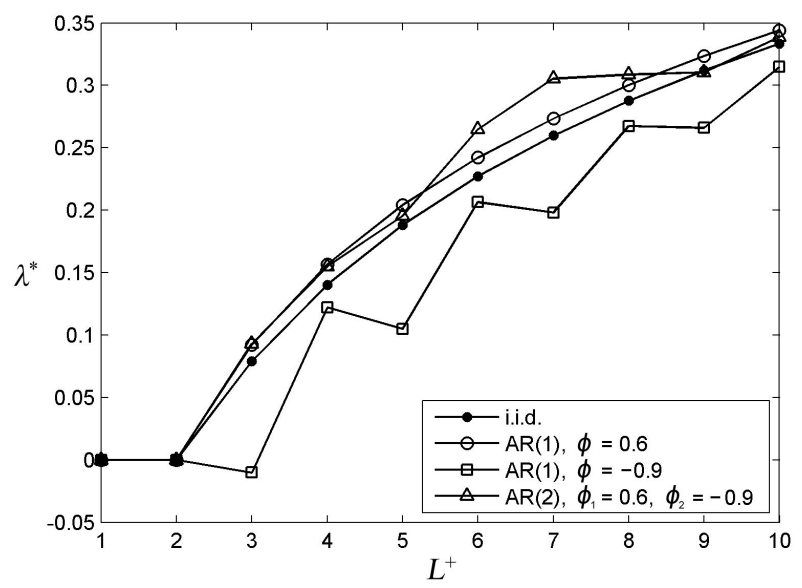

Fig. 5. The relationship between $\lambda^{*}$ and $L^{+}$when lead-time follows a uniform distribution

Lastly, we have calculated the optimal proportional policy under a real-life lead-time distribution shown in Figure 1, with demand following a range of ARMA processes. Table IV shows the result. Once again we see the ability of the proportional policy to reduce order and inventory variances simultaneously. However due to the relatively low lead-time variability and demand correlation, such benefit is sometimes less significant.

\section{CONCLUSION AND DisCUSSION}

Based on our personal experience of observing a stochastic lead time in practice, we studied its effect on system variances with correlated demand. Given a known (or perceived) lead time distribution and demand correlation information, we presented a state-space approach to calculate the inventory variance and proved that the proportional policy outperforms 
TABLE IV

OPTIMAL FEEDBACK CONTROLLER AND SYSTEM VARIABILITY WITH A REAL-LIFE LEAD TIME DISTRIBUTION

\begin{tabular}{|c|c|c|c|c|c|c|c|}
\hline \multirow{2}{*}{ Demand type } & \multicolumn{2}{|c|}{ OUT $(1-\lambda=1)$} & \multicolumn{3}{|c|}{ POUT } & \multirow{2}{*}{$\begin{array}{l}\% \Sigma_{i i} \\
\text { Reduction }\end{array}$} & \multirow{2}{*}{$\begin{array}{l}\% \Sigma_{o o} \\
\text { Reduction }\end{array}$} \\
\hline & $\Sigma_{i i}$ & $\Sigma_{o o}$ & $1-\lambda^{*}$ & $\Sigma_{i i}$ & $\Sigma_{o o}$ & & \\
\hline i.i.d. & 14.28 & 1 & 0.92 & 14.27 & 0.84 & 0.07 & 15.87 \\
\hline $\mathrm{AR}(1), \phi=0.6$ & 20.59 & 4.96 & 0.91 & 20.55 & 4.24 & 0.19 & 14.52 \\
\hline $\mathrm{AR}(1), \phi=-0.9$ & 13.21 & 0.28 & 0.96 & 13.21 & 0.27 & 0.00 & 3.57 \\
\hline $\operatorname{AR}(2), \phi_{1}=0.6, \phi_{1}=-0.9$ & 16.94 & 1.49 & 0.95 & 16.94 & 1.48 & 0.01 & 0.29 \\
\hline $\operatorname{ARMA}(1,1), \phi=0.6, \theta=-0.9$ & 37.63 & 16.60 & 0.91 & 37.48 & 14.26 & 0.40 & 14.10 \\
\hline
\end{tabular}

the classical order-up-to policy in reducing both order and inventory variations.

The impact of order crossover has been revealed. Ref. [9] and [10] previously showed that when the probability of order crossover is zero, the distribution of the shortfall and that of the lead time demand is identical. We went on to show that the optimality of order-up-to policy is also contingent on order crossover rather than on the randomness of lead time. We have shown that when order crossover is present, the classical order-up-to policy is no longer optimal at minimizing inventory variance. When order crossover is not present (e.g., when lead time follows a two-point distribution with adjacent values), the classical order-up-to policy is optimal at reducing inventory fluctuation. However it is worth pointing out that in most cases stochastic lead time does infer order crossover.

This finding directly leads to the conclusion that the proportional policy is able to achieve reduce inventory related costs and smooth orders simultaneously, which have long been considered as conflicting objectives [23] [17]. The extent of order smoothing depends on the value of optimal proportional controller, which is further related to the lead-time distribution and demand correlation. Numerical investigation shows that the benefit of the proportional policy is more obvious when the lead-time is more variable, e.g., when it follows a uniform distribution or a nonadjacent two-point distribution, and when demand is positively correlated. When the variability of lead-time is low, the order smoothing effect will be limited. When there is a strong negative correlation between successive demands and $L^{+}$is small, the trade-off between order and inventory variance may reappear. But the benefit of the proportional policy remains in the majority of cases we have investigated.

In Table $\mathrm{V}$ we summarize the properties of the inventory system under conditions of constant lead-time, stochastic lead-time without crossover and stochastic lead-time with crossover. It can be concluded that the stochasticity of lead-time affects the inventory distribution, and hence the relationship between average demand and inventory variance.
Under stochastic lead-time, the density function of inventory becomes multi-modal, and the demand level becomes a significant contributor to inventory variation. On the other hand, order crossover determines whether the classical orderup-to policy is the proper choice for minimizing inventory variance and inventory related costs. With order crossover, the proportional order-up-to policy reduces inventory variance. Given that the order variance decreases with $\lambda$, this also reduces the order variance.

Managerially this result has interesting consequences. One of the concerns of practitioners have when adopting a proportional policy in their replenishment decisions is its potential aggravating effect to the inventory related cost, as Lemma 3.1 dictates. However, our finding suggests that, under order crossover scenarios (which are quite common in practice), this concern may not be necessary since inventory variance can be reduced by proportional control (albeit marginally), and this subsequently allows one to reduce order variance considerably. Even if the proportional controller is not set optimally due to statistical and computational imperfections, the benefit of reducing bullwhip will arguably outweigh the relatively small loss of increased inventory cost. The intuition is that the bullwhip is always sensitive to the proportional controller, but inventory variance will be less sensitive, especially when the mean demand is large and lead-time is highly variable. Therefore we conclude that the proportional policy is increasing desirable with volatile delays and positively correlated demand.

This research can be further developed in the following directions. An exogenous lead time distribution is assumed throughout this paper for simplicity. However in practice businesses may be able to adjust transportation mode (and hence the lead-time) based on system states. For example in times of low stock or high demand, air freight could be used to expedite replenishments. Thus it will be intriguing to incorporate dependence of transportation delay on system states. From the theoretical perspective, a thorough understanding of this problem calls for more knowledge on the mathematical properties of the objectives, e.g. monotonicity and convexity of 
TABLE V

SUMMARIZATION OF THE PROPERTIES OF DIFFERENT LEAD-TIME CONDITIONS

\begin{tabular}{|c|c|c|c|}
\hline & Constant lead-time & $\begin{array}{l}\text { Stochastic lead-time } \\
\text { without crossover }\end{array}$ & \multicolumn{1}{|c|}{$\begin{array}{c}\text { Stochastic lead-time } \\
\text { with crossover }\end{array}$} \\
\hline Shape of inventory pdf & The function is bell-shaped & \multicolumn{2}{|c|}{ The function is multi-modal } \\
\hline Relationship of $\bar{d}$ and $\Sigma_{i i}$ & $\begin{array}{l}\text { Inventory variance is independent } \\
\text { of average demand levels }\end{array}$ & Inventory variance is dependent upon average demand levels \\
\hline Optimal Policy for $\Sigma_{i i}$ & Order-up-to policy minimizes inventory variance & $\begin{array}{l}\text { Proportional policy minimizes } \\
\text { inventory variance }\end{array}$ \\
\hline Relationship of $\Sigma_{o o}$ and $\Sigma_{i i}$ & Minimizing inventory variance increases bullwhip & $\begin{array}{l}\text { Minimizing inventory variance } \\
\text { decreases bullwhip }\end{array}$ \\
\hline
\end{tabular}

the order and inventory variances w.r.t. $\lambda$ for ARMA demand and stochastic lead-times.

\section{REFERENCES}

[1] H. Lee, V. Padmanabhan, and S. Whang, "Information distortion in a supply chain: The bullwhip effect," Management Science, vol. 43, pp. 546-558, 1997.

[2] F. Chen, Z. Dresner, J. Ryan, and D. Simchi-Levi, "Quantifying the bullwhip effect in a simple supply chain: The impact of forecasting, lead times, and information," Management Science, vol. 46, pp. 436443, 2000.

[3] C. Holt, F. Modigliani, and H. Simon, "A linear decision rule for production and employment scheduling," Management Science, vol. 2, pp. 1-30, 1955.

[4] P. Zipkin, "Stochastic lead times in continuous-time inventory models," Naval Research Logistics Quarterly, vol. 33, pp. 763-774, 1986.

[5] J. Riezebos, "Inventory order crossovers," International Journal of Production Economics, vol. 104, pp. 666-675, 2006.

[6] J. Hayya, U. Bagchi, J. Kim, and D. Sun, "On static stochastic order crossover," International Journal of Production Research, vol. 114, pp. 404-413, 2008.

[7] G. Hadley and T. Whitin, Analysis of Inventory Systems. Englewood Cliffs, NJ.: Prentice-Hall, 1963.

[8] J.-S. Song, "The effect of lead time uncertainty in a simple stochastic inventory model," Management Science, vol. 40, pp. 603-613, 1994.

[9] D. Zalkind, "Order-level inventory systems with independent stochastic lead times," Management Science, vol. 24, pp. 1384-1392, 1978.

[10] L. Robinson, J. Bradley, and L. Thomas, "Consequences of order crossover under order-up-to inventory policies," Manufacturing and Service Operations Management, vol. 3, pp. 175-188, 2001.

[11] J. Bradley and L. Robinson, "Improved base-stock approximations for independent stochastic lead times with order crossover," Manufacturing and Service Operations Management, vol. 7, pp. 319-329, 2005.

[12] P. Finch, "The order cycle system of stock control," Journal of the Australian Mathematical Society, vol. 2, pp. 209-216, 1961.

[13] N. Agin, "A min-max inventory model," Management Science, vol. 12, pp. 517-529, 1966.

[14] U. Bagchi, J. Hayya, and C.-H. Chu, "The effect of lead time variability: the case of independent demand," Journal of Operations Management, vol. 6, pp. 159-177, 1986

[15] D. Chatfield, J. Kim, T. Harrison, and J. Hayya, "The bullwhip effect - impact of stochastic lead time, information quality, and information sharing: a simulation study," Production and Operations Management, vol. 13, pp. 340-353, 2004.

[16] J. Kim, D. Chatfield, T. Harrison, and J. Hayya, "Quantifying the bullwhip effect in a supply chain with stochastic lead time," European Journal of Operational Research, vol. 173, pp. 617-636, 2006.

[17] S. Disney, D. Towill, and W. Van de Velde, "Variance amplification and the golden ratio in production and inventory control," International Journal of Production Economics, vol. 90, pp. 295-309, 2004.

[18] G. Gaalman, "Bullwhip reduction for ARMA demand: The proportional order-up-to policy versus the full-state-feedback policy," Automatica vol. 42, pp. 1283-1290, 2006.

[19] Y. Chen and S. Disney, "The myopic order-up-to policy with a proportional feedback controller," International Journal of Production Research, vol. 45, pp. 351-368, 2007.
[20] G. Gaalman and S. Disney, "On bullwhip in a family of order-up-to policies with $\operatorname{ARMA}(2,2)$ demand and arbitrary lead times," International Journal of Production Economics, vol. 121, pp. 454-463, 2009.

[21] S. Disney, L. Hoshiko, L. Polley, and C. Weigel, "Removing bullwhip from lexmarks toner operations," in Proceedings of Production and Operations Management Society Annual Conference, Denver, USA, May 2013.

[22] J. Magee, "Guides to inventory control part II," Harvard Business Review, vol. 34, pp. 103-116, 1956.

[23] D. Deziel and S. Eilon, "A linear production-inventory control rule," The Production Engineer, vol. 43, pp. 93-104, 1967.

[24] D. Towill, "Dynamic analysis of an inventory and order based production control system," International Journal of Production Research, vol. 20, pp. 671-687, 1982.

[25] K. Åström, Introduction to Stochastic Control Theory. New York: Academic Press, 1970. 